\title{
NONSINGULAR RINGS
}

FRANCIS L. SANDOMIERSKI ${ }^{1}$

1. Introduction. This paper is intended to extend some well-known results for integral domains to nonsingular rings and to give a characterization of right-semihereditary rings using their maximal right quotient rings.

For homological notions the reader is referred to [2]. For the notions, singular submodule, finite dimensional module, the reader is referred to [7], [8]. For the notion of maximal quotient ring see [5].

For a module $M_{R}, Z\left(M_{R}\right)$ denotes the singular submodule.

All rings are rings with identity and all modules are unitary.

2. Semihereditary and hereditary rings. Gentile [6] has shown that if $R$ is a ring such that every principal right ideal is projective, then $Z\left(R_{R}\right)=0$ and if $R$ is commutative and every principal ideal is $R$-flat, then $Z(R)=0$, hence right (left) semihereditary rings have zero singular right (left) ideal.

Theorem 2.1. If $R$ is a ring such that $Z\left(R_{R}\right)=0$ and $P_{R}$ is an $R$ projective module containing a finitely generated large submodule, then $P_{R}$ is finitely generated.

Proof. By [2, Proposition VII, 3.1], there exists a family $\left\{x_{\alpha}\right\}$ of elements of $P_{R}$ and a family $\left\{f_{\alpha}\right\} \subseteq_{R} P^{*}=\operatorname{Hom}\left(P_{R}, R_{R}\right)$ such that for all $x \in P, x=\sum x_{\alpha} f_{\alpha}(x)$, where $f_{\alpha}(x)=0$ for all but a finite number of $\alpha$.

Clearly, it is sufficient to show that $f_{\alpha}$ is the zero map for all but finitely many $\alpha$.

Let $B_{R}$ be a finitely generated large submodule of $P_{R}$, with generators $b_{1}, \cdots, b_{n}$, then $\left\{\alpha \mid f_{\alpha}\left(b_{i}\right) \neq 0\right.$ for some $\left.1 \leqq i \leqq n\right\}=A$ is a finite set of the indices and $f_{\alpha}(B)=0$, if $\alpha \notin A$. If $x \in P$, then $I=\{r \in R \mid x r \in B\}$ is a large right ideal of $R$ by [10, Proposition 1.2] and for $\alpha \notin A, 0=f_{\alpha}(x r)=f_{\alpha}(x) r$ for every $r \in I$, so $f_{\alpha}(x) \in Z\left(R_{R}\right)=0$ and the theorem follows.

CoROllaRy 1. If $R$ is a ring such that $Z\left(R_{R}\right)=0$ and $d\left(R_{R}\right)$ is finite, then every $R$-projective right ideal is finitely generated.

Proof. If $I_{R}$ is an $R$-projective right ideal of $R$, then $I_{R}$ contains as a large submodule of $I_{R}$ a finitely generated right ideal, by [10, Theorem 1.3], hence by the theorem $I_{R}$ is finitely generated.

Received by the editors November 22, 1966.

1 This paper was written with partial support from NSF Grant GP-3993. 
COROLLARY 2. If $R$ is a right hereditary ring with $d\left(R_{R}\right)$ finite, then $R$ is right Noetherian.

Proof. Since $R$ is right hereditary $Z\left(R_{R}\right)=0$ by remark in the Introduction. By Corollary 1 , every $R$-projective right ideal is finitely generated, so $R$ is right Noetherian since every right ideal is projective.

Corollary 1 generalizes the result that over a commutative integral domain every projective ideal is finitely generated and Corollary 2 generalizes the result that a commutative integral domain which is a hereditary ring is Noetherian.

The notion of a closed submodule as considered in [8] for example will be useful.

Definition 2.2. A submodule $B_{R}$ of a right $R$-module $A_{R}$ is closed in $A$ if $B$ has no proper essential extension in $A$, i.e. if $C$ is a submodule containing $B$ with $B$ large in $C$, then $B=C$.

Lemma 2.3. (a) If $B$ is a submodule of $A$ such that $Z(A / B)=0$, then $B$ is closed in $A$.

(b) If $B$ is closed in $A$ and $Z(A)=0$, then $Z(A / B)=0$.

Proof. (a) If $C$ is an essential extension of $B$ in $A$ and $x \in C$, then $I=\{r \in R \mid x r \in B\}$ is a large right ideal of $R, b y$ [10, Proposition 1.2], hence $x+B \in Z(A / B)=0$, so $x \in B$ and it follows that $B$ is closed in $A$.

(b) If $x+B \in Z(A / B)$, then $I=\{r \in R \mid x r \in B\}$ is a large right ideal of $R$. The claim is that $B+x R$ is an essential extension of $B$. If $y=b$ $+x t \in B+x R$ with $b \in B, t \in R$, then $J=\{r \in R \mid t r \in I\}$ is a large right ideal of $R$. Since $Z(A)=0$, there is an $r \in J$ such that $y r \neq 0$, but $y r \in B$, so $B$ is a large submodule of $B+x R$. Since $B$ is closed, $x \in B$, so $x+B=0$ and (b) follows.

The proofs of the results in the following proposition can be found in [8].

Proposition 2.4. (a) If $M_{R}$ is a finite dimensional right $R$-module and $K_{R}$ is a closed submodule of $M$, then $M / K$ is finite dimensional and $d(M)=d(M / K)+d(K)$ (where $d(M)=\operatorname{dim}(M)$ of [8]).

(b) If $M_{R}=M_{1} \oplus \cdots \oplus M_{n}$ is the direct sum of the finite dimensional submodules $M_{j}, j=1,2, \cdots, n$, then $M$ is finite dimensional and $d(M)=\sum_{j=1}^{m} d\left(M_{j}\right)$.

Lemma 2.5. If $R$ is a finite dimensional right $R$-module and $M_{R}$ is a finitely generated nonsingular right $R$-module, then $M_{R}$ is finite dimensional.

Proof. $M$ is an epimorphic image of a finitely generated free right 
$R$-module $F_{R}$ with kernel $K_{R}$. By Proposition 2.4(b), $F_{R}$ is of finite dimension. Since $M$ is isomorphic to $F / K, F / K$ is nonsingular, so by Lemma 2.3(a), $K$ is a closed submodule of $F$. Now by Proposition 2.4(a), $F / K$ is finite dimensional, hence so is $M$.

THEOREM 2.6. If $R$ is right semihereditary and finite dimensional as a right $R$-module, then every finitely generated torsionless [1] right $R$ module is $R$-projective.

Proof. Let $M_{R}$ be a finitely generated torsionless right $R$-module. Since $R$ is semihereditary, $Z\left(R_{R}\right)=0$, hence it is clear that $Z\left(M_{R}\right)=0$. since $M$ is contained in a direct product of copies of $R_{R}$. By Lemma 2.5 , it follows that $M_{R}$ is finite dimensional.

Since $M$ is torsionless there is $0 \neq f \in \in_{R} M^{*}=\operatorname{Hom}\left(M_{R}, R_{R}\right)$. Since $\operatorname{Im}(f)$ is a finitely generated right ideal of $R, K=\operatorname{Ker}(f)$ is a direct summand of $M$, because $\operatorname{Im}(f)$ is $R$-projective. Let $M=K \oplus I$. Now proceed by induction on $d(M)=n$, dimension of $M$, [8]

If $n=1$, then $K=0$ since $f \neq 0$, so $M$ is isomorphic to $\operatorname{Im}(f)$, hence $R$ projective. If $K=0$, then $M_{R}$ is $R$-projective. If $K \neq 0$, then $K$ is finitely generated torsionless, and $d(K)<d(M)$ since $\operatorname{Im}(f) \cong I \neq 0$, hence by induction, $K$ is $R$-projective and the theorem follows.

COROLlaRy. If $R$ is right semihereditary and finite dimensional as a right $R$-module, then $R$ is left semihereditary.

Proof. In view of Chase [3, Theorem 4.1] it is sufficient to show that every torsionless right $R$-module is $R$-flat. Since submodules of torsionless modules are torsionless and the functors $\operatorname{Tor}_{n}^{R}$ commute with direct limits, it is sufficient to show that a finitely generated torsionless right $R$-module is $R$-flat, because a module is the direct limit of its finitely generated submodules. The corollary follows from the theorem since $R$-projective modules are $R$-flat.

Theorem 2.7. Let $Q$ be a ring such that $Q$ is right $Q$-injective, then every finitely generated nonsingular right $Q$-module is $Q$-projective and $Q$-injective.

Proof. Let $M$ be a finitely generated nonsingular right $Q$-module. Then $M$ is the epimorphic image of a free right $Q$-module $F$ with finite basis. Let $K$ denote the kernel of the epimorphism. Since $F$ is free on a finite basis, $F$ is $Q$-injective. Since $M$ is nonsingular $K$ is closed in $F$. Clearly a closed submodule of an injective module is a direct summand, hence it follows that $M$ is $Q$-projective and $Q$-injective.

Lemma 2.8. If $R$ is a ring with $Z\left(R_{R}\right)=0$ and $0 \rightarrow K \rightarrow F \rightarrow M \rightarrow 0$ a 
short exact sequence of right $R$-modules such that $F$ is free, $K$ contains a finitely generated large submodule $L$ and $M$ is $R$-flat, then $M$ is $R$-projective.

Proof. Let $u_{1}, \cdots, u_{n}$ be generators of the finitely generated large submodule $L$ of $K$. Since $M$ is $R$-flat by [3, Proposition 2.2] there is an $R$-homomorphism $\theta: F \rightarrow K$ such that $\theta$ is the identity map on $L$. If $x \in K$, then $I=\{r \in R \mid x r \in L\}$ is a large right ideal of $R$. For $r \in I$, $x r=\theta(x r)=\theta(x) r$ so $(x-\theta(x)) r=0$ for each $r \in I$, hence $x-\theta(x)$ $\in Z(F)=0$ since $Z\left(R_{R}\right)=0$. Thus, it is shown that $\theta$ is the identity on $K$, so the short exact sequence splits and the lemma follows.

THEOREM 2.8. Let $R$ be a ring with $Z\left(R_{R}\right)=0, Q$ its maximal right quotient ring and $M_{R}$ a finitely generated right $R$ module. Then $M_{R}$ is $R$-projective if and only if $M_{R}$ is $R$-flat and $M \otimes_{R} Q$ is Q-projective.

Proof. There is an exact sequence $0 \rightarrow K_{R} \rightarrow F_{R} \rightarrow M_{R} \rightarrow 0$ where $F_{R}$ is free on a finite basis, $K$ is the kernel of the epimorphism $F \rightarrow M$ and $K \rightarrow F$ is the inclusion map.

Only if: If $M_{R}$ is $R$-projective, then the sequence splits and therefore the sequence

$$
0 \rightarrow K \otimes_{R} Q \rightarrow F \otimes_{R} Q \rightarrow M \otimes_{R} Q \rightarrow 0
$$

of right $Q$-modules is split exact. Since $F \otimes_{R} Q$ is a free right $Q$-module, it follows that $M \otimes_{R} Q$ is right $Q$-projective.

If: In view of Lemma 2.8 it is sufficient to show that $K$ contains a finitely generated large submodule. Since $M_{R}$ is $R$-flat and $M \otimes_{R} Q$ is $Q$-projective, the sequence $0 \rightarrow K \otimes Q \rightarrow F \otimes Q \rightarrow M \otimes Q \rightarrow 0$ is split exact and it follows that $K \otimes Q$ is a finitely generated right $Q$-module. Clearly a finite set of generators of $K \otimes Q$ as a right $Q$-module of the form $k_{i} \otimes 1, i=1, \cdots, n$ with $k_{i} \in K$ exists. Let $L$ be the submodule of $K$ generated by $k_{1}, \cdots, k_{n}$. Since $K$ is nonsingular, the sequence $0 \rightarrow K \rightarrow K \otimes Q$ is exact by [10, Proposition 2.2], $K$ may be identified in $K \otimes Q$ with those elements of the form $x \otimes 1, x \in K$. If $0 \neq x \in K$, then $x \otimes 1=\sum_{i=1}^{n} k_{i} \otimes q_{i}$ with $q_{i} \in Q$. Let $I=\left\{r \in R \mid q_{i} r \in R\right.$ for all $\left.i\right\}$, then by [10, Proposition 1.2], $I$ is a large right ideal of $R$. Clearly, $K \otimes Q$ is nonsingular as a right $R$-module since $Q_{R}$ is, there is an $r \in I$ such that $\left.0 \neq x r \otimes 1=(x \otimes 1) r=\sum_{i} k_{i} \otimes q_{i} r=\sum_{i} k_{i}\left(q_{i} r\right)\right) \otimes 1$ and it follows that $0 \neq x r \in L$ since $\sum_{i} k_{i}\left(q_{i} r\right) \in L$, so $L$ is a large submodule of $K$ and the if part follows.

COROLlaRy 1. If $R$ is a ring with $Z\left(R_{R}\right)=0$ and $R_{R}$ is finite dimensional, then every finitely generated flat right $R$-module is $R$-projective.

Proof. In this case, $Q$, the maximal right quotient ring of $R$, is 
semisimple with minimum condition, by [10, Theorem 2.3 ], so every $Q$-module is $Q$-projective and the corollary follows.

A special case of Corollary 1 is

Corollary 2. If $R$ is a commutative integral domain, then every finitely generated $R$-flat $R$-module is projective.

Endo [4], has also established Corollary 2 as a consequence of a more general theorem for commutative rings and their total quotient rings. However, Corollary 2 can be proven directly, without appealing to any quotient rings, using Lemma 2.8.

TheOREM 2.9. Let $R$ be a ring with $Z\left(R_{R}\right)=0, Q$ its maximal quotient ring such that ${ }_{R} Q$ is left $R$-flat. Then every finitely generated $R$-flat submodule of a free right $R$-module is $R$-projective.

Proof. Let $M_{R}$ be a finitely generated submodule of the free right $R$-module $F_{R}^{\prime}$. Since ${ }_{R} Q$ is left $R$-flat the sequence of right $Q$-modules $0 \rightarrow M \otimes_{R} Q \rightarrow F \otimes_{R} Q$ is exact, hence it follows that $M \otimes_{R} Q$ is a nonsingular right $Q$-module since $Q$ is. Clearly, $M \otimes_{R} Q$ is a finitely generated right $Q$-module and $Q$ is right $Q$-injective module, by Theorem 2.7, $M \otimes_{R} Q$ is $Q$-projective. If $M_{R}$ is $R$-flat, then by Theorem 2.8 $M_{R}$ is $R$-projective.

It is well known, e.g. [3], that a commutative integral domain $R$ is semihereditary (Prüfer ring) if and only if $\operatorname{GWD}(R) \leqq 1$. Chase [3] generalized this for arbitrary semihereditary rings. Another generalization will be given here.

THEOREM 2.10. For a ring $R$ the following statements are equivalent.

(1) $R$ is right semihereditary.

(2) $Z\left(R_{R}\right)=0$. GWD $(R) \leqq 1$ and $Q$ the maximal right quotient ring of $R$ is left $R$-flat.

Proof. (1) implies (2). $Z\left(R_{R}\right)=0$ by the remark at the beginning of this paper and that $\operatorname{GWD}(R) \leqq 1$ is well known, e.g. [2]. To show that ${ }_{R} Q$ is left $R$-flat it is sufficient to show that if $I$ is a finitely generated right ideal of $R$, then the sequence $0 \rightarrow I \otimes_{R} Q \rightarrow R \otimes_{R} Q$ is exact. Since $I$ is $R$-projective, by Theorem $2.8, I \otimes_{R} Q$ is $Q$-projective, hence nonsingular as a right $R$-module. If $\sum_{i=1}^{n} a_{i} \otimes q_{i}=0$ in $R \otimes_{R} Q$, with $a_{i} \in I, q_{i} \in Q$, then $\sum_{i} a_{i} q_{i}=0$ and $J=\left\{r \in R \mid q_{i} r \in R\right.$ all $\left.i\right\}$ is a large right ideal in $R$ by [10, Proposition 1.2]. In $I \otimes_{R} Q$, for $r \in J, \quad\left(\sum_{i} a_{i} \otimes q_{i}\right) r=\sum a_{i} \otimes q_{i} r=\left(\sum_{i} a_{i}\left(q_{i} r\right)\right) \otimes 1=0 \otimes 1=0, \quad$ since $\sum_{i} a_{i} q_{i}=0$, so $\sum_{i} a_{i} \otimes q_{i} \in Z(I \otimes Q)=0$ and (2) follows.

(2) implies (1). If $I_{R}$ is a finitely generated right ideal of $R$, then $I_{R}$ is $R$-flat since GWD $(R) \leqq 1$, so by Theorem $2.9 I_{R}$ is $R$-projective and (1) follows. 


\section{REFERENCES}

1. H. Bass, Finitistic dimension and a homological generalization of semiprimary rings, Trans. Amer. Math. Soc. 95 (1960), 466-488.

2. H. Cartan and S. Eilenberg, Homological algebra, Princeton Univ. Press, Princeton, N. J., 1956.

3. S. U. Chase, Direct products of modules, Trans. Amer. Math. Soc. 97 (1960), $457-473$.

4. S. Endo, On flat modules over commutative rings, J. Math. Soc. Japan 14 (1962), 284-291.

5. G. D. Findlay and J. Lambek, A generalized ring of quotients. I, II, Canad. Math. Bull. 1 (1958), 77-85, 155-167.

6. E. R. Gentile, Singular submodule and injective hull, Indag. Math. 24 (1962), 426-433.

7. A. W. Goldie, The structure of prime ring under ascending chain conditions, Proc. London Math. Soc. (3) 8 (1958), 589-608.

8. - Semiprime rings with maximum condition, Proc. London Math. Soc. (3) 10 (1960), 201-220.

9. - - Torsion-free modules and rings, J. Algebra 1 (1964), 268-287.

10. F. Sandomierski, Semisimple maximal quotient rings, Trans. Amer. Math. Soc. 128 (1967), 112-120.

UNIVERSITY OF Wisconsin 